\title{
Prevalence and risk factors associated with cat parasites in Italy: a multicenter study
}

\author{
Marco Genchi ${ }^{1}$, Alice Vismarra' ${ }^{1}$ Stefania Zanet ${ }^{2}$, Simone Morelli ${ }^{3}$, Roberta Galuppi ${ }^{4}$, Giuseppe Cringoli ${ }^{5}$, \\ Riccardo Lia ${ }^{6}$, Manuela Diaferia ${ }^{7}$, Antonio Frangipane di Regalbono ${ }^{8}$, Giulia Venegoni ${ }^{9}$, Fabrizio Solari Basano ${ }^{9}$, \\ Antonio Varcasia ${ }^{10}$, Stefania Perrucci ${ }^{11}$, Vincenzo Musella ${ }^{12}$, Emanuele Brianti ${ }^{13}$, Alessia Gazzonis ${ }^{14}$, \\ Michele Drigo ${ }^{15}$, Liliana Colombo ${ }^{16}$ and Laura Kramer ${ }^{1 *}$
}

\begin{abstract}
Background: Parasites that infect cats include protozoa, helminths and arthropods, many of which are transmissible to humans. Effective control relies on a good knowledge of parasite distribution and the risk factors for infection. The present study was aimed at evaluating the prevalence of major feline parasites in Italy and the risk factors associated with their occurrence.

Methods: Over a 12-month study period, feces, hair and ectoparasites from naturally infected cats from feral colonies, shelters and private households were analyzed at 13 study centers across Italy. Samples from these cats $(n=987)$ were analyzed at all centers using the same diagnostic methods. Prevalence values and risk factors were evaluated statistically for the identification of predictors of risk.

Results: The overall prevalence of gastro-intestinal and broncho-pulmonary (BP) nematodes was 35.9\% (354/987). Toxocara cati was the most prevalent species (253/987; 25.6\%), followed by Ancylostomatidae (98/987; 9.9\%). Among BP nematodes, Aelurostrongylus abstrusus was the most common (76/987; 7.7\%). Approximately 35.7\% (352/987) of the study population was infested by ectoparasites, of which the most common were fleas (29.4\%, 290/987), followed by ear mites Otodectes cynotis $(9.8 \%, 97 / 987)$. Predictors of risk for parasite infection included age, a predominantly or exclusively outdoor lifestyle, geographic area and lack of antiparasitic treatment.

Conclusions: Both ecto- and endoparasites are still common in cats throughout Italy, many of them being of zoonotic concern and vectors of pathogens to humans. Given the presence of parasites throughout the entire study period, year-round treatment should be considered. Furthermore, data confirm the need to protect the human-animal bond using proper endo- and ectoparasiticides to reduce the risk of human infection, in application of the OneHealth concept.
\end{abstract}

Keywords: Cat, Parasites, Italy, Prevalence, Zoonosis, Risk factors

*Correspondence: kramerlh@unipr.it

1 Dipartimento Di Scienze Medico-Veterinarie, Università Di Parma, via del Taglio, 10, 43126 Parma, Italy

Full list of author information is available at the end of the article

\section{Background}

Numerous parasites infect domestic cats. Among these, helminths of the gastro-intestinal (GI) tract and respiratory system can cause severe disease if parasite loads are heavy, while different arthropods can cause skin disease and allergy. Subclinical infection is of equal concern given the zoonotic nature of several feline helminths and the capacity of fleas and ticks to transmit pathogens to cats, original author(s) and the source, provide a link to the Creative Commons licence, and indicate if changes were made. The images or other third party material in this article are included in the article's Creative Commons licence, unless indicated otherwise in a credit line to the material. If material is not included in the article's Creative Commons licence and your intended use is not permitted by statutory regulation or exceeds the permitted use, you will need to obtain permission directly from the copyright holder. To view a copy of this licence, visit http://creativecommons.org/licenses/by/4.0/. The Creative Commons Public Domain Dedication waiver (http://creativeco mmons.org/publicdomain/zero/1.0/) applies to the data made available in this article, unless otherwise stated in a credit line to the data. 
other animals and humans. Recent multicenter studies in both Europe and Italy have been carried out to define the current status of endo- and ectoparasite infections of cats [1-4]. The results of these studies indicate that infections are widespread and depend on various risk factors, including lifestyle, geographical area and frequency of antiparasitic treatment. Most studies on prevalence, distribution and risk factors for feline parasites in Italy have been carried out in the central and southern areas of the country $[5,6]$.

Multicenter studies provide useful information on the distribution and risk of parasite infection. However, few apply the same, standard diagnostic protocols at each center, thus compromising comparability of the results. Indeed, it has been reported that different copromicroscopic techniques have differing sensitivity, specificity and accuracy for the diagnosis of GI and broncho-pulmonary (BP) nematodes.

Seasonality of parasite infection in cats has been evaluated mostly in retrospective, longitudinal studies [12, 13]. It is possible that sampling and analysis over a fixed period of time may provide more useful information on the current effects of season on parasite prevalence.

The aims of the present multicenter study were therefore to: (i) determine the current prevalence of feline endo- and ectoparasites throughout Italy through the recruitment of cats from all regions; (ii) evaluate seasonal trends by recruiting a set number of cats each month consecutively over a 12-month period; (iii) use standardized diagnostic methods in order to eliminate variables associated with differences in test sensitivities/specificities/accuracy; (iv) identify those factors that significantly increase the risk of infection.

\section{Methods}

\section{Animals and study period}

The study period was from July 2019 to September 2020.

Thirteen university study centers participated, and each center had a recruitment target of seven cats per month for 12 months, for a total of 84 cats per center. Each cat could only be included in the study once, with no more than two cats from the same home/shelter/colony sampled. Randomization was based on the target number of seven cats: once the target number was reached, enrollment ceased even if there were other cats that met the inclusion criteria.

\section{Enrollment and sample collection}

Inclusion criteria included: outdoor access; no antiparasitic treatment (endo and/or ecto) in the 3 months prior to enrollment; and signed informed consent. Exclusion criteria included: no outdoor access; and antiparasitic treatment (endo and/or ecto) in the 3 months prior to enrollment.

Each study center was supplied with tubes $(15 \mathrm{ml})$ prefilled with $80 \%$ ethanol; flea combs and zip-lock bags; one Mini-FLOTAC kit Fill-FLOTAC [14] containing four Mini-FLOTAC and four Fill-FLOTAC devices (200 tests); and instructions for material collection, conservation and analyses.

At enrollment, general information, inclusion criteria, clinical observations, frequency of antiparasitic treatments in the previous 12 months and eventual signs of ectoparasitic infestation were recorded, and an online data collection sheet was filled in (Additional file 1: Text S1). Any observed ticks, lice and nits were collected (mites were collected by scrapings or ear wax collection while nits, lice and ticks were simply removed with tweezers) and stored in the provided 15-ml tubes containing $80 \%$ ethanol. Each cat was combed with a flea comb for 5 min, and collected material was stored in the zip-lock plastic bag at $4{ }^{\circ} \mathrm{C}$. Owners were asked to submit at least $7 \mathrm{~g}$ of fresh feces, which were collected, examined for the eventual presence of proglottids and stored at $4{ }^{\circ} \mathrm{C}$.

\section{Laboratory analyses}

All collected material was analyzed at the university laboratory of each participating center.

Material collected with flea combs was examined under a stereomicroscope and the presence of flea/flea debris recorded. Feces were examined for the presence of proglottids and identified according to Soulsby et al. [15].

Mini-FLOTAC copromicroscopic examination was carried out on $2 \mathrm{~g}$ of feces in $18 \mathrm{ml}$ of $\mathrm{NaCl}$ floating solution (specific gravity: 1.200) according to the protocol described in Cringoli et al. [7]. Minimum/maximum number of eggs/oocysts/cysts per gram of feces (EPG/ OPG/CPG) were calculated. The Baermann test was carried out on $5 \mathrm{~g}$ of feces and the feces examined approximately $12 \mathrm{~h}$ later, according to Bowman et al. [16]. Larvae were identified according to Varcasia et al. [17] and Brianti et al. [18].

Study center reference personnel were asked to register with the Castor $^{\circledR}$ EDC $^{\circledR}$ database [19], which was managed by the study monitor. Each reference personnel had her/his own login credentials. Results were transcribed into the database, preferably within several days of receipt and analyses.

\section{Statistical analyses}

Chi-square tests were carried out to evaluate the association between positivity for at least one endo-/ectoparasite infection, Toxocara cati, Ancylostomatidae, Aelurostrongylus abstrusus, fleas and Otodectes cynotis, as well as for the following categorical variables: sex, geographical 
area (North, Central, South), lifestyle (exclusive outdoor/ predominantly outdoor/predominantly indoor), provenance (privately owned, shelter, colony), age ( $<1$ year, 1-5 years, $>5$ years) and antiparasitic treatment in the previous year (yes/no).

Relationships between infection and the variables ("predictors") were analyzed by multivariable regression analysis applying a stepwise backward elimination method (IBM SPSS Statistics for Macintosh, version 27.0; IBM Corp., Armonk, NY, USA.). Statistical significance was set at $\alpha=0.05$.

\section{Results}

\section{Study population}

Severe restrictions on movement/activity were put into place to contain the SARS-CoV-2 pandemic (March 2020 "lockdown"), which resulted in a decrease in/lack of cat enrollment at many participating centers from March 2020 to May 2020, leading to an extension of the study for a further 2 months. A total of 987 cats were enrolled.

Cats were evenly distributed in terms of sex. Approximately half the study population (47.3\%) was within the age range of $1-5$ years, with the remaining cats evenly distributed between $<1$ year $(28.1 \%)$ and $>5$ years of age (24.6\%). Over $40 \%$ of enrolled cats were from southern Italy and $69.2 \%$ were privately owned cats. Over $75.8 \%$ of the study population lived predominantly outdoor/exclusively outdoor (Table 1 ).

Most cats, including privately owned animals, had not received antiparasitic treatment in the year before enrollment. Overall, 539 of the 987 (54.6\%) cats in the study were infected with one or more parasites. Of these 987 infected animals, $13.7 \%(n=135)$ had at least two endoparasites and $7.4 \%$ (73) had at least two arthropod infestations.

\section{Endoparasites}

The overall prevalence of GI and BP nematodes was $35.9 \%$ (354/987). Toxocara cati was the most prevalent species (253/987; 25.6\%), followed by Ancylostomatidae (98/987; 9.9\%). Among the BP nematodes, Aelurostrong abstrusus was the most common (76/987; 7.7\%), while Capillaria aerophila (Eucoleus aerophilus; 22/987, $2.2 \%)$ and Troglostrongylus brevior (12/987, 1.2\%) were much less frequently found (Fig. 1; Table 2). Among the GI protozoa identified, Cystoisospora felis was the most common $(101 / 987 ; 10.2 \%)$ while all other species were uncommon (Fig. 1; Table 2). Based on macroscopic examination of proglottids [15], members of class Cestoda were rarely found (Dipylidium caninum 3.3\%, Taenia taeniaeformis 1.3\%, Mesocestoides spp. 0.1\%) (Fig. 1; Table 2).
Table 1 Characteristics of the study population

\begin{tabular}{lclc}
\hline Characteristics & Values, $n(\%)$ & Characteristics & Values, $n(\%)$ \\
\hline Sex & & Number of ectoparasiticide \\
& & treatments done in the last year \\
Male & $492(49.8)$ & 0 & $655(66.4)$ \\
Female & $495(50.2)$ & 1 & $192(19.5)$ \\
Status & & 2 & $59(6.0)$ \\
Non-sterilized & $438(44.4)$ & 3 & $38(3.9)$ \\
Sterlized & $549(55.6)$ & 4 & $24(2.4)$ \\
Provenance & & 5 & $7(0.7)$ \\
Shelter & $29(2.9)$ & 6 & $11(1.1)$ \\
Colony & $275(27.9)$ & 7 & $0(0.0)$ \\
Privately owned & $683(69.2)$ & 8 & $1(0.1)$ \\
Lifestyle & & Number of endoparasiticide \\
& & treatments done in the last year \\
Predominantly indoor & $239(24.2)$ & 0 & $752(76.2)$ \\
Predominatly outdoor & $423(42.9)$ & 1 & $159(16.1)$ \\
Exclusively outdoor & $325(32.9)$ & 2 & $47(4.8)$ \\
Geographical area in Italy & 3 & $14(1.4)$ \\
North & $331(33.5)$ & 4 & $10(1.0)$ \\
Central & $223(22.6)$ & 5 & $1(0.1)$ \\
South & $433(43.9)$ & 6 & $3(0.3)$ \\
Age (years) & $277(28.1)$ & 8 & $0(0.0)$ \\
$<1$ & $467(47.3)$ & & $1(0.1)$ \\
1-5 & $243(24.6)$ & & \\
$>$ 5 & & & \\
\hline & & 7 &
\end{tabular}

Of the $35.9 \%$ cats found to be positive for any GI or BP nematodes, $20.1 \%$ were female and $15.8 \%$ were male. Of the female cats analyzed, $40.0 \%$ were positive for endoparasite infection, while $31.7 \%$ of the male cats analyzed showed endoparasites; this difference was statistically significant $\left(X^{2}=7.37, d f=1, P=0.007\right)$. In addition, when compared to males, females had significantly higher prevalence values for $T$. cati $\left(28.5\right.$ vs $22.8 \% ; X^{2}=4.23, d f=1$, $P=0.04)$ and Ancylostomatidae (13.5 vs 6.3\%; $X^{2}=14.44$, $d f=1, P<0.001$.

Colony cats had a significantly higher prevalence of endoparasite infections (45.5\%; $X^{2}=15.25, d f=2$, $P<0.001)$ compared to both shelter $(31.0 \%)$ and privately owned cats (32.2\%). Infection with $A$. abstrusus and Ancylostomatidae was significantly higher in colony and shelter cats (15.3 and $10.3 \%$, respectively; $X^{2}=$ $13.90, d f=2, P<0.001$ ) compared to privately-owned cats (4.5\%), while the prevalence of $T$. cati infection was comparable among the colony/shelter/privately owned cats.

Overall prevalence of endoparasite infection was significantly higher in cats aged $<1$ year $\left(49.8 \% ; X^{2}=\right.$ 56.95, $d f=1, P<0.001)$ and in cats aged between 1 and 5 years of age $\left(36.8 \% ; X^{2}=56.95, d f=1, P<0.001\right)$, with T. cati infection significantly more frequent in cats 


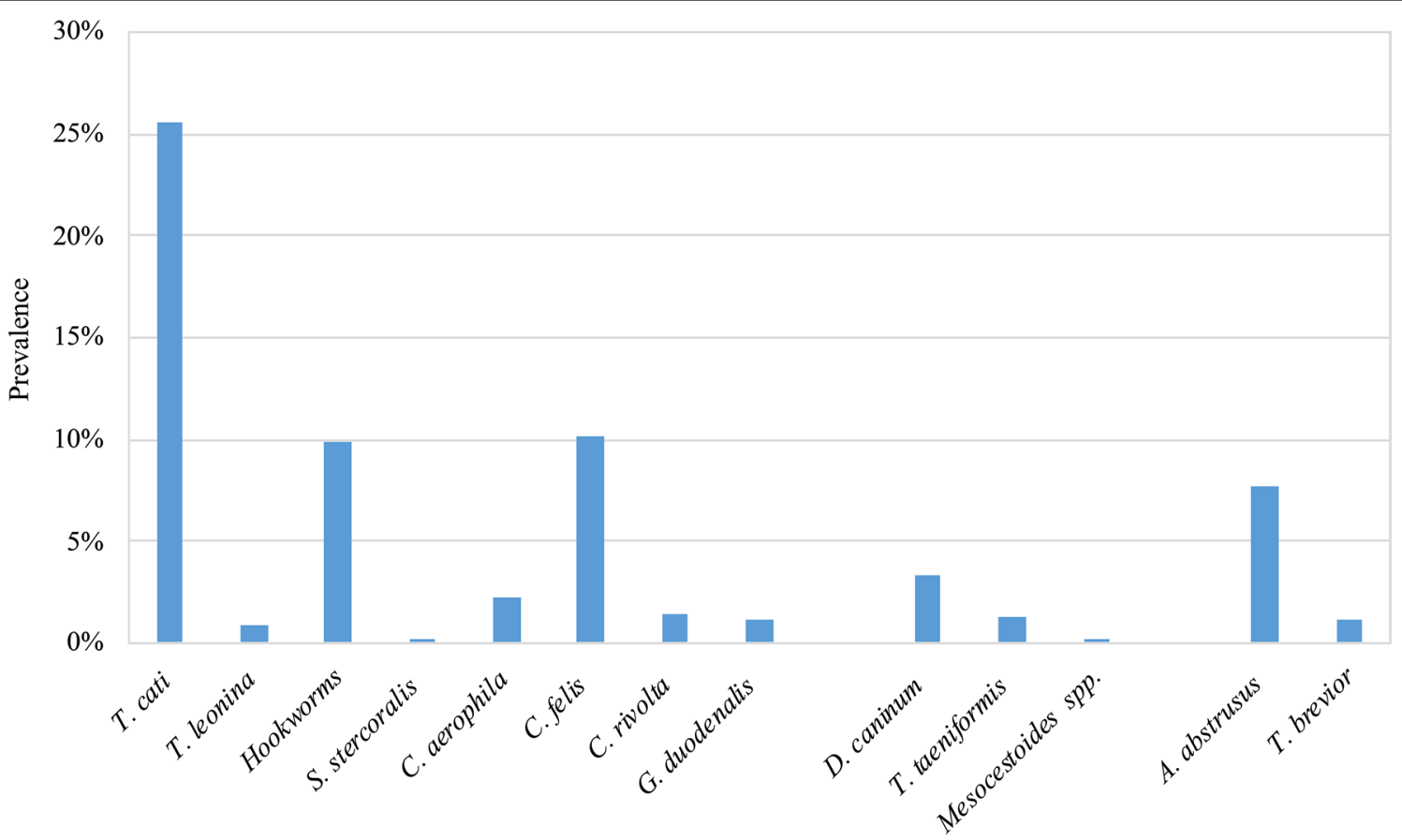

Fig. 1 Overall prevalence of gastro-intestinal and broncho-pulmonary endoparasites

aged $<1$ year $\left(42.6 \% ; X^{2}=80.68, d f=1, P<0.001\right)$ than in the other age groups (1-5 years: $24.6 \%$; $>5$ years: $8.2 \%)$. Infection with Ancylostomatidae was significantly more frequent in cats aged between 1 and 5 years $(12.4 \%$; $\left.X^{2}=6.39, d f=1, P=0.041\right)$ than in cats in the other age categories. Prevalence of $A$. abstrusus was significantly higher in cats aged $<1$ year $(9.7 \%)$ and between 1 and 5 years $\left(8.6 \% ; X^{2}=7.58, d f=1, P=0.023\right)$. Coccidiosis was more prevalent in younger cats $(20.6 \%)$.

Prevalence of endoparasite infection was directly associated with the frequency of outdoor access. Cats with an exclusively outdoor lifestyle had significantly higher infection rates for $A$. abstrusus $\left(14.5 \% ; X^{2}=33.01, d f=2\right.$, $P<0.001)$ compared to cats living predominantly outdoors or predominantly indoors (5.4 and 2.5\%, respectively). Cats with a predominantly/exclusively outdoor lifestyle had significantly higher prevalence values for $T$. cati $\left(X^{2}=12.87, d f=2, P=0.02\right)$ and Ancylostomatidae $\left(X^{2}=29.73, d f=2, P<0.001\right)$. Cats residing in the areas of southern Italy had significantly higher prevalence values for $T$. cati $\left(X^{2}=23.84, d f=2, P<0.001\right)$, Ancylostomatidae $\left(X^{2}=37.34, d f=2, P<0.001\right)$ and $A$. abstrusus $\left(X^{2}=33.59, d f=2, P<0.00\right)$.

Monthly prevalence throughout the study was variable (Fig. 2), but there was no month in which parasites were not observed.

Minimum/maximum EPG/OPG/CPG results for helminths and protozoa were as follows: T. cati (from 5 up to 7,50,000 EPG), T. leonina (from 10 up to 950 EPG), Ancylostomatidae (from 5 up to 8495 EPG), C. aerophila (from 5 to 300 EPG), C. felis (from 10 up to 21,200 OPG), C. rivolta (from 5 up to 21,200 OPG), and G. duodenalis (from 10 up to 550 CPG).

\section{Ectoparasites}

A total of $35.7 \%(352 / 987)$ of the study population was infested by ectoparasites. The most common parasites were fleas (Ctenocephalides felis felis; 29.4\%, 290/987), followed by ear mites (O. cynotis; 9.8\%, 97/987). Tick infestation (Ixodes spp.: 3.12\%; Rhipicephalus spp.: 0.18\%) was uncommon, as was infestation by other mites and lice [i.e. Notoedres:5/987, 0.5\%; Neotrombicula:2/987, 0.2\%; Cheyletiella: $1 / 987,0.1 \%$; lice (unidentified): 8/987, 0.8\%] (Fig. 3; Table 2).

Of the total cat population analyzed, prevalence values for ectoparasites were comparable between male (17.3\%) and female (18.3\%) cats. Of the 492 females examined, $36.6 \%$ were infested, and of 495 males examined, 34.8\% had ectoparasites. Overall prevalence of flea infestation for the 987 cats recruited was $14.5 \%$ in females versus $14.9 \%$ in males; for O. cynotis infestation, this was $5.7 \mathrm{ver}$ sus $4.2 \%$. Considering gender specifically, 28.9 and $11.3 \%$ of examined female cats were infested with fleas and $O$. cynotis, respectively, and 29.9 and $8.3 \%$, respectively, of male cats were infested with these two ectoparasites. 


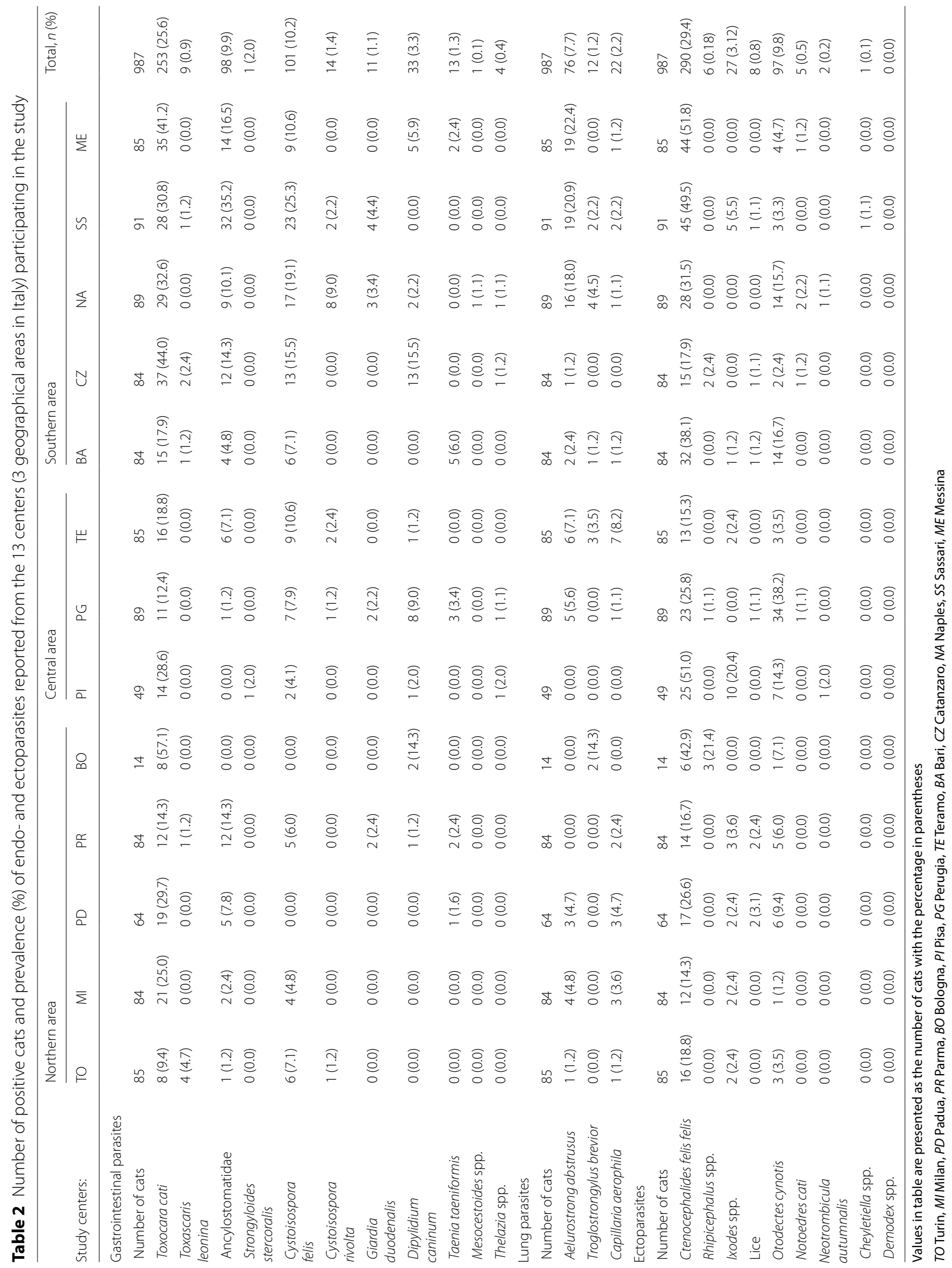



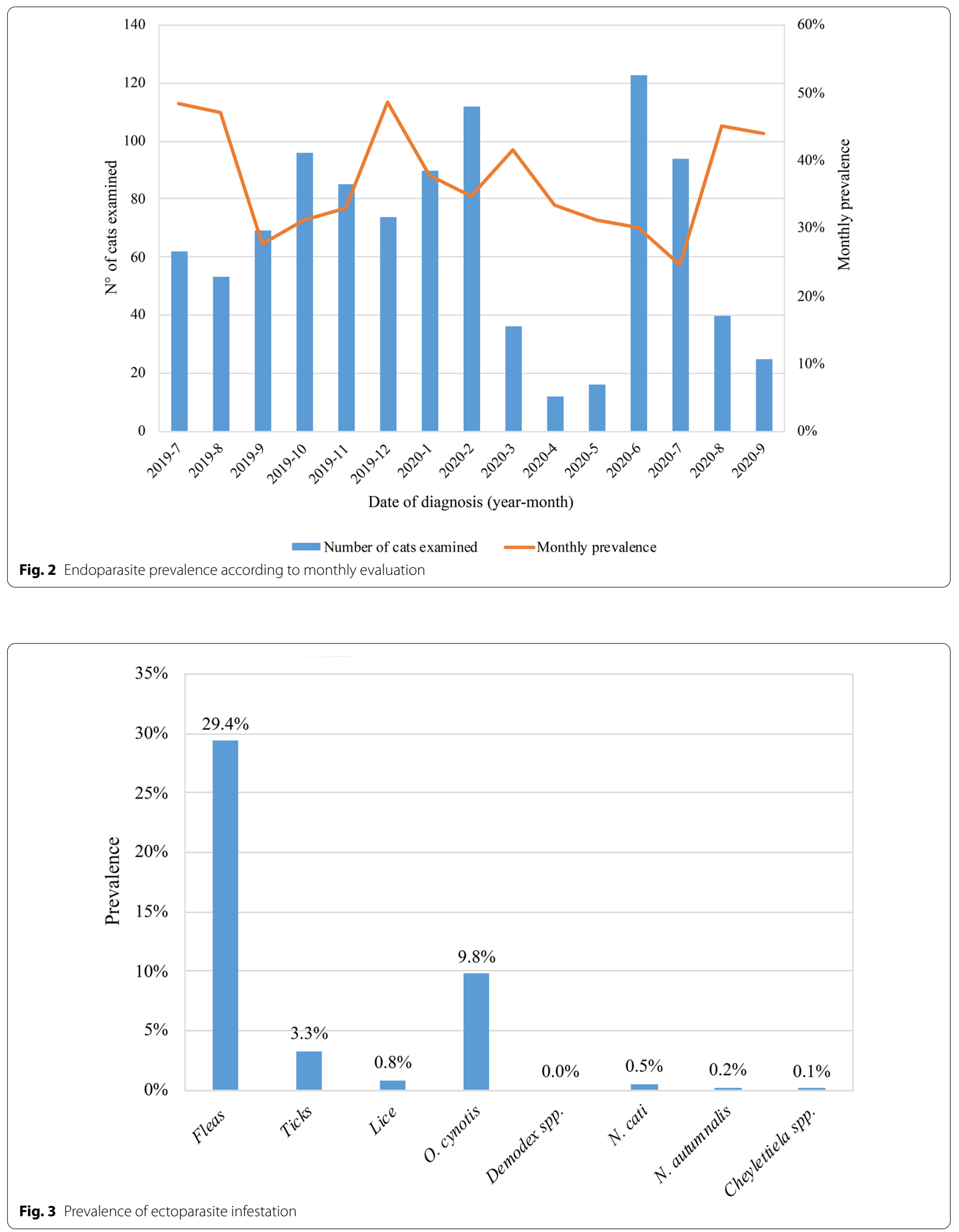


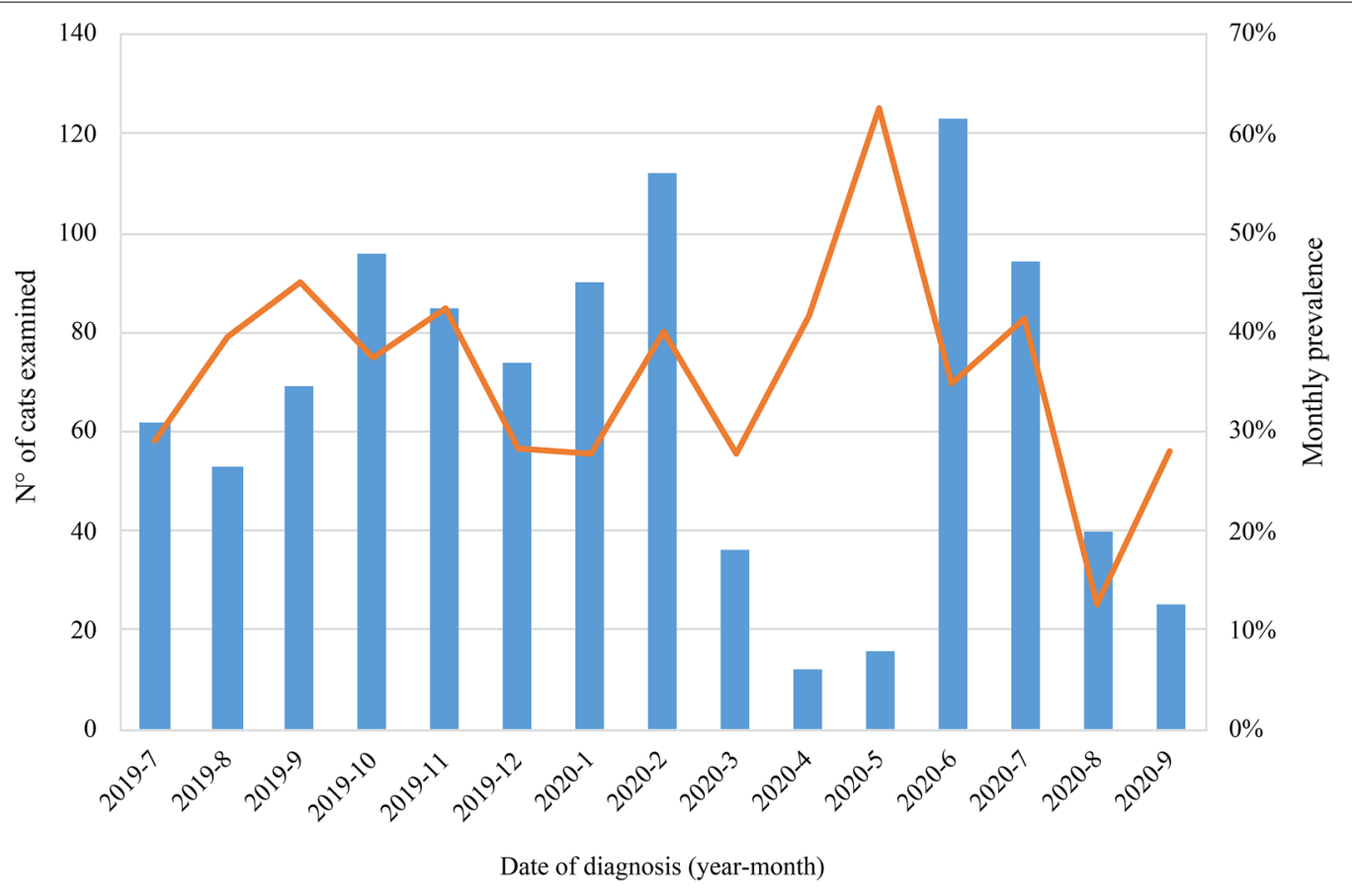

Number of cats examined

Monthly prevalence

Fig. 4 Monthly prevalence of ectoparasite infestations

Compared to shelter and privately owned cats, colony cats had a significantly higher overall prevalence of ectoparasites $\left(53.5 \%\right.$ vs 31.0 and $28.7 \%$, respectively; $X^{2}$ $=52.65, d f=2, P<0.001)$, fleas $(46.5 \%$ vs 24.1 and $22.7 \%$, respectively; $\left.X^{2}=54.15, d f=2, P<0.001\right)$ and ear mites (17.5\% vs 3.4 and $7.8 \%$, respectively; $X^{2}=25.42, d f=2$, $P<0.001)$. The same was observed for cats with a predominantly/exclusively outdoor lifestyle [35.5 and 51.4\%, respectively, vs $14.6 \%$ (indoor lifestyle); $X^{2}=81.03, d f=2$, $P<0.001]$.

Overall prevalence for ectoparasite infestation was significantly higher in cats aged $<1$ year $(45.8 \%)$ and between 1 and 5 years $(35.5 \%)\left(X^{2}=26.25, d f=1, P<0.001\right)$ compared to cats aged $>5$ years $(24.3 \%)$. Flea infestation was significantly more prevalent in cats aged $<1$ year $\left(36.5 \% ; X^{2}=15.80, d f=1, P<0.001\right)$ and between 1 and 5 years $\left(29.8 \% ; X^{2}=15.80, d f=1, P<0.001\right)$ than in older cats $(20.6 \%)$. The same was observed for O. cynotis, which showed significantly higher prevalence in cats aged $<1$ year $\left(12.3 \% ; X^{2}=7.77, d f=1, P=0.02\right)$ and between 1 and 5 years $\left(10.7 \% ; X^{2}=7.77, d f=1, P=0.02\right)$ (cats $>5$ years: $5.3 \%$ ).

The prevalence of ectoparasites was significantly higher in cats from central (37.7\%) and southern (42.7\%) Italy (compared to northern area) (25.1\%) $\left(X^{2}=25.97, d f=2\right.$, $P<0.001)$. Interestingly, the prevalence of $O$. cynotis infestation was significantly higher $\left(19.7 \% ; X^{2}=34.80\right.$, $d f=2, P<0.001)$ in cats from central Italy than in those from the northern (4.8\%) and southern (8.5\%) areas.

Monthly prevalence throughout the study was variable (Fig. 4). Only $23.8 \%$ of the cats had received at least one treatment against endoparasites in the past year, while only $33.6 \%$ of the cats had received treatment against ectoparasites in the past year.

\section{Risk factors for endo-ectoparasites infestation}

Significant risk factors as determined from univariate analysis were entered in the multivariable logistic regression model in order to address possible confounding factors and to compute adjusted odds ratios (OR). Tables 3 and 4 report the results of the multivariate analysis, which considered overall prevalence of endoparasite infection, overall prevalence of ectoparasite infestation and prevalence of the most common endoparasites ( $T$. cati, Ancylostomatidae and $A$. abstrusus) and ectoparasites (fleas and O. cynotis) as dependent variables and sex, age, provenance, lifestyle, geographical area and anti-parasitic treatment as predictors. The results highlight that age was predictive for $T$. cati infection in cats aged $<1$ year [OR 7.834, 95\% confidence interval (CI) 4.609-13.314] and between 1-5 years (OR 3.382, 95\% CI 22.021-5.661), but not for Ancylostomatidae or A. abtrusus. Outdoor lifestyle put cats at higher risk for all three nematodes $(T$. cati: OR 2.659, 95\% CI 1.622-4.361; Ancylostomatidae: 
Table 3 Multivariable regression analyses for endoparasites

\begin{tabular}{lll}
\hline Significant predictor of risk & $P$ value & OR (95\% Cl) \\
\hline Endoparasite infection & & \\
Female & 0.047 & $1.331(1.003-1.765)$ \\
Age (<1 year) & 0.000 & $3.953(2.587-6.041)$ \\
Age (1-5 year) & 0.000 & $2.198(1.483-3.258)$ \\
Exclusively outdoor & 0.000 & $3.515(2.223-5.560)$ \\
South & 0.000 & $2.052(1.476-2.853)$ \\
No treatment & 0.018 & $1.517(1.074-2.143)$ \\
Toxocara cati & & \\
Age (<1 year ) & 0.000 & $7.834(4.609-13.314)$ \\
Age (1-5 year ) & 0.000 & $3.382(2.021-5.661)$ \\
Exclusively outdoor & 0.000 & $2.659(1.622-4.361)$ \\
South & 0.018 & $1.542(1.076-2.208)$ \\
Ancylostomatidae & & \\
Female & 0.001 & $2.218(1.390-3.537)$ \\
Predominantly outdoor & 0.012 & $3.144(1.285-7.691)$ \\
South & 0.000 & $3.277(1.874-5.730)$ \\
No treatment & 0.006 & $2.472(1.300-4.700)$ \\
Aelurostrongylus abstrusus & & \\
Exclusively outdoor & 0.010 & $3.558(1.354-9.354)$ \\
South & 0.000 & $5.480(2.498-12.024)$ \\
No treatment & 0.020 & $2.440(1.154-5.160)$ \\
\hline
\end{tabular}

Multivariable regression analyses were performed using IBM SPSS Statistics for Macintosh, version 27.0

Statistical significance was set at $\alpha=0.05$

$\mathrm{Cl}$ Confidence interval, $O R$ odds ratio

OR 3.144, 95\% CI1.285-7.691; A. abstrusus OR 3.558, 95\% CI 1.354-9.354). Significant predictors for ectoparasite infestations included living in a colony (OR 1.612, 95\% CI1.114-2.334) and an exclusive (OR 4.497, 95\% CI2.764-7.318) or predominantly (OR 3.197 95\% CI 2.084-4.905) outdoor lifestyle.

\section{Discussion}

The study provides a overview of the endo- and ectoparasites affecting Italian cats during a 15-month study period. Despite monthly recruitment being brusquely interrupted due to the SARS-CoV-2 pandemic, preventing several centers from reaching the established number of cats per month, nearly 1000 cats were analyzed. Importantly, laboratory analyses were carried out according to standardized protocols which were followed by all centers, thus reducing the risk of variability associated with different test sensitivities/specificities. It should be noted, however, that while using one method does improve comparability, having different people use the method can influence results.

The present study also applied univariate and multivariate analyses to evaluate overall risk for endoparasites or ectoparasites and for the most common helminths and arthropods observed.

Our findings show that feline endo- and ectoparasites are widespread in Italy, with varying prevalence across the different regions. Approximately 55.0\% (539/987) of cats enrolled in our study were infected with at least one internal or external parasite. This compares well with the results of a study at the European level [1] which reported that more than half $(50.7 \%)$ of the cats studied were infected with one or more endo- and/or ectoparasite.

Among the GI helminths, T. cati was the most frequently found (25.6\% of enrolled cats). This prevalence is higher than that reported by a previous Italian multicenter study [4] (21.6\%), and is also higher than the mean European prevalence value reported in 2017 by Giannelli et al. [2] (14.5\%). The prevalence is, however, in line with that of other surveys conducted in Italy $[5,6]$. In the present study, risk for $T$. cati infection included age ( $<5$ years of age), an exclusively outdoor lifestyle and living in southern Italy. Pre- and perinatal transmission and age immunity are well known for Toxocara spp., while outdoor access favors exposure to both highly resistant eggs contaminating the environment and to paratenic hosts [20]. The warmer climatic conditions in southern Italy and Italian islands likely increase the presence and persistence of infective stages of the parasite [21].

Ancylostomatidae represented the second most frequent group of nematodes diagnosed in the study population (9.9\%). In a study performed at the European level [2], the mean prevalence of hookworms was reported to be $4.5 \%$, while in some selected areas of Italy the prevalence was reported to be $4.9 \%$ [4]. In the present study, Ancylostomatidae infection was associated with gender (females were at increased risk), predominantly outdoor access, living in southern Italy and lack of anthelmintic treatment. Age was not a significant risk predictor. Indeed, it is assumed that there is no transmammary transmission of Ancylostomatidae from the queen to her kittens [22].

As mentioned above, warmer climatic conditions in southern Italy may increase the presence and persistence of infective stages of Ancylostomatidae. For transmission to occur, Ancylostomatidae need to develop into infective larvae in the soil from eggs passed in the host's stool, and higher temperature and humidity (tropical and subtropical climates) provide an adequate environment for this growth stage [23].

The feline lungworm A. abstrusus was present in $7.7 \%$ of the cats analyzed in our study; in comparison, Giannelli et al. [2] reported a mean prevalence in Europe of $8.2 \%$, while recent data from Italy [4] reported a prevalence of $10.3 \%$. Multivariate analysis indicated that an exclusively outdoor lifestyle, living in southern Italy and 
lack of anthelmintic treatment were significant risk factors for A. abstrusus. Outdoor access has been reported previously as an important risk factor for A. abstrusus [24]. While the geographical distribution of feline lungworms tends to be patchy but stable in endemic hotspots [2], interpretation of geographical location as a risk factor should be done with caution, and any geographic location reporting autochthonous circulation of the parasite should be considered to be potentially endemic. Traversa et al. [4] reported a 20.0\% prevalence of $A$. abstrusus in Piedmont, while in the present study only $1.2 \%$ of cats were infected. Giannelli et al. [2] reported that $16.7 \%$ of cats from the province of Bari were infected with $A$. abstrusus, compared to a prevalence of $2.4 \%$ in our study. Values from the provinces of Sassari (SS) and Messina (ME), on the other hand, were higher in the present study (20.9 and $22.4 \%$, respectively) (Table 2), compared to data reported by Giannelli et al. [2] (11.6 and 15.3\%, respectively), but lower than the prevalence of $25.2 \%$ reported by Tamponi et al. [25] in a previous study in Sardinia. Interestingly, univariate analysis showed that cats aged $>5$ years had a significantly higher prevalence for $A$. abstrusus, but age was not confirmed as a risk factor following multivariate evaluation. As expected, larvae of $T$. brevior were found mostly in cats from southern regions. Nevertheless, it is worth mentioning that, in the present study, this potentially fatal metastrongyloid was also found in two cats in northern Italy, indicating an apparent northward geographical expansion.

The Mini-FLOTAC technique [7] has been recently demonstrated to be a highly sensitive method for diagnosing parasitic infections of human and veterinary importance where larvae or ova of parasites are shed in the feces [8-11]. Moreover, the mini-FLOTAC technique in combination with Fill-FLOTAC has been shown to be user-friendly and safe, with a wide diagnostic range. These features are particularly useful for monitoring and control programs in which large numbers of fecal samples need to be processed rapidly and safely. In the present study, the harmonized use of the Mini-FLOTAC technique allowed the qualitative and quantitative analysis of parasite load without the need for specialized equipment.

Fleas were the most common ectoparasite found in the present study (29.4\%). Cooper et al. [26] reported similar prevalence values in a recent nationwide study in the UK. Beugnet et al. [1] reported prevalence values ranging from $3.6 \%$ in Bari to $31.4 \%$ in Naples. Multivariate analysis showed that infestation was associated with young age ( $<1$ year), living in a colony, predominantly/exclusively outdoor lifestyle, living in central and southern Italy and lack of ectoparasitic treatment. Cooper et al. [27] reported geographical differences (prevalence declining
Table 4 Multivariable analyses for ectoparasites

\begin{tabular}{lll}
\hline Significant predictor of risk & $P$ value & OR (95\% Cl) \\
\hline Ectoparasite infection & & \\
Age (<1 year) & 0.000 & $2.290(1.518-3.455)$ \\
Colony & 0.011 & $1.612(1.114-2.334)$ \\
Predominantly outdoor & 0.000 & $3.197(2.084-4.905)$ \\
Exclusively outdoor & 0.000 & $4.497(2.764-7.318)$ \\
Central & 0.001 & $1.962(1.320-2.916)$ \\
South & 0.001 & $1.757(1.256-2.458)$ \\
Fleas & & \\
Age (<1 year) & 0.017 & $1.699(1.099-2.626)$ \\
Colony & 0.049 & $1.459(1.001-2.125)$ \\
Predominantly outdoor & 0.000 & $3.294(2.024-5.361)$ \\
Exclusively outdoor & 0.000 & $5.092(2.974-8.718)$ \\
Central & 0.030 & $1.606(1.046-2.465)$ \\
South & 0.000 & $2.069(1.448-2.958)$ \\
No treatment & 0.002 & $1.725(1.215-2.449)$ \\
Otodectes cynotis & & \\
Age (<1 year) & 0.017 & $2.377(1.164-4.854)$ \\
Colony & 0.000 & $3.232(1.801-5.803)$ \\
Predominantly outdoor & 0.030 & $2.193(1.077-4.462)$ \\
Central & 0.000 & $4.526(2.652-7.724)$ \\
\hline
\end{tabular}

Multivariable regression analyses were performed using IBM SPSS Statistics for Macintosh, version 27.0

Statistical significance was set at $a=0.05$

from south to north in UK) and no treatment as significant predictors of risk for flea infestation. Beugnet et al. [1] identified outdoor access as the only risk factor in a multivariate analysis.

The ear mite O. cynotis was present in $9.8 \%$ of enrolled cats. Beugnet et al. [1] reported prevalence values of $40.3 \%$ in cats from Bari and $21.8 \%$ in cats from the area of Naples. In Tuscany (central Italy), O. cynotis was identified in $66.1 \%$ cats with otitis externa [27]. Age $<1$ year, living in a colony, having a predominantly outdoor lifestyle and coming from central Italy were all factors identified as increasing the risk of ear mite infestation in cats. Otodectes cynotis is transmitted by direct contact and is highly contagious. Young cats are more playful and likely have more direct contact with other cats.

The main limitation of the present study is the potential effect of bias, based on inclusion criteria. While on the one hand cats that had received antiparasitic treatment in the 3 months preceding enrollment were excluded, the evaluation of the effect of treatment versus no treatment was carried out considering the frequency of treatment for the 12 months prior to enrollment (see Additional file 1: Text S1). Beugnet et al. had a similar study design [1], including cats that had received no treatment in the 2 months prior to 
enrollment and analyzing the frequency of antiparasitic treatment as a risk factor for infection. Outdoor access was another enrollment bias. Indeed, this has already been identified as a risk factor for parasite infection in cats [1-3]. In the present study, however, access was categorized as infrequent, frequent or exclusive. The analysis of risk was based on the frequency of access, not on outdoor versus indoor.

\section{Conclusions}

The results of this study highlight the fact that both ectoand endoparasites are still common in cats throughout Italy. Interestingly, of the 239 cats with a predominantly indoor lifestyle, $31.8 \%$ were affected by endo- and ectoparasites, suggesting that parasiticide treatment is more important than lifestyle. Therefore, taking the zoonotic implications and the clinical importance into account, it is strongly advisable to promote effective and regular parasite control in cats, with adequate frequencies of treatment for both internal and external parasites. It is interesting to note that there was no month in which endo- and ectoparasites could not be found, suggesting that cats can be infected throughout the year. This would imply that parasite infection should not be considered seasonal, but that control should be year-round. However, only $23.8 \%$ of the cats had received at least one treatment against endoparasites in the past year, while only $33.6 \%$ of the cats had received treatment against ectoparasites in the past year. The European Council for the Control of Companion Animal Parasites (ESCCAP) recommends "year-round, life-long" parasite control. The aim of the present study was not to associate climate and environmental conditions to risk of parasite infection, but rather to support these recommendations. Furthermore, many privately owned cats spend a significant amount of time outdoors where they are exposed to parasites. Practitioners need to inform their clients of the risks and recommend routine antiparasitic treatment. Interestingly, southern Italy continues to show higher prevalence of parasite infection in cats. This may be due to climatic, social or economic factors, and practitioners working in these areas should be particularly attentive.

Finally, zoonotic parasites and vectors of human disease are still widespread in cats, confirming the need to protect the human-animal bond and the application of the One-Health concept.

\section{Abbreviations}

BP: Broncho-pulmonary; Gl: Gastro-intestinal nematodes.

\section{Supplementary Information}

The online version contains supplementary material available at https://doi. org/10.1186/s13071-021-04981-2.

Additional file 1: Text S1. Study Form 2 Enrollment.

\section{Acknowledgements}

The authors wish to thank Dr. Chiara Cattabiani for assisting with parasitological analyses in Parma; Dr. Silvia Carta, Dr. Antonio Viglietti, Dr. Paola Fois and Prof. Antonio Scala for collaborating at sampling and parasitological analysis in Sardinia; Dr. Antonio Bosco and Dr. Lavinia Ciuca (University of Napoli).

\section{Authors' contributions}

Study technical manager and monitor: Arcoblu srl, Via Alessandro Milesi, 5, 20,133 Milan (ref. FSB). Study coordinators: MG and LK, Dept. di Scienze Medico-Veterinarie, Università degli Studi di Parma, via del Taglio 10, 43,126, Parma.

Experiments were conceived and designed by all authors. Sampling was done by all authors. Experiments were performed by all authors. The data were analyzed by MG and FSB. Statistical analyses were performed by FSB. The manuscript was written by LK, MG and AV and critically revised by all authors. All authors read and approved the final manuscript.

\section{Funding}

This study was funded by MSD-AH srl, Via Flli. Cervi snc, Centro Direzionale Milano Due, Palazzo Canova, 20090 Segrate Milano (ref. Liliana Colombo).

\section{Availability of data and materials}

All data generated or analyzed during this study are included in this published article.

\section{Declarations}

\section{Ethics approval and consent to participate}

The cats' owners signed an informed consent authorizing the use of faeces and hair for the study.

\section{Consent for publication}

Informed consent was obtained from all individual participants included in the study.

\section{Competing interests}

The authors declare that they have no competing interests.

\section{Author details}

${ }^{1}$ Dipartimento Di Scienze Medico-Veterinarie, Università Di Parma, via del Taglio, 10, 43126 Parma, Italy. ${ }^{2}$ Dipartimento Di Scienze Veterinarie, Università Di Torino, L.Go Braccini, 2, 10095 Grugliasco, TO, Italy. ${ }^{3}$ Facoltà Di Medicina Veterinaria, Università Degli Studi Di Teramo, 64100 Teramo, Località Piano d'Accio, Italy. ${ }^{4}$ Dipartimento Di Scienze Mediche Veterinarie, Università Di Bologna, Via Tolara di Sopra, 50, Ozzano Emilia, 40064 Bologna, Italy. ${ }^{5}$ Dipartimento Di Medicina Veterinaria E Produzioni Animali, Università Di Napoli Federico II, Via Federico Delpino 1, 80137 Naples, Italy. ${ }^{6}$ Dipartimento Di Medicina Veterinaria, Università Degli Studi Di Bari, Valenzano, 70010 Bari, Italy. ${ }^{7}$ Dipartimento Di Medicina Veterinaria, Università Degli Studi Di Perugia, Via San Costanzo 4, 06126 Perugia, Italy. ${ }^{8}$ Dipartimento Di Medicina Animale, Produzioni E Salute, Università Degli Studi Di Padova, Viale dell'Università, 16, 35020 Legnaro, PD, Italy. ${ }^{9}$ Arcoblu S.R.L, Via Alessandro Milesi 5, 20133 Milan, Italy. ${ }^{10}$ Dipartimento Di Medicina Veterinaria, Università Degli Studi Di Sassari, Via Vienna 2, 07100 Sassari, Italy. ${ }^{11}$ Dipartimento Di Scienze Veterinarie, Università Degli Studi Di Pisa, Viale delle Piagge 2, 56124 Pisa, Italy. ${ }^{12}$ Dipartimento Di Scienze Della Salute, Università Di Catanzaro Magna Graecia, 88100 Catanzaro, Italy. ${ }^{13}$ Dipartimento Di Scienze Veterinarie, Università Degli Studi Di Messina, 98168 Messina, Italy. ${ }^{14}$ Dipartimento Di Medicina Veterinaria, Università Degli Studi Di Milano, via dell'Università 6, 26900 Lodi, Italy. ${ }^{15}$ Dipartimento Di Medicina Animale, Produzioni E Salute, Università Degli Studi Di Padova, Viale dell'Università, 16, 35020 Legnaro, PD, Italy. ${ }^{16}$ MSD Animal Health, Via Fratelli Cervi, 20090 Segrate, MI, Italy. 
Received: 24 May 2021 Accepted: 26 August 2021

Published online: 15 September 2021

\section{References}

1. Beugnet F, Bourdeau P, Chalvet-Monfray K, Cozma V, Farkas R, Guillot J, et al. Parasites of domestic owned cats in Europe: co-infestations and risk factors. Parasit Vectors. 2014;7:291.

2. Giannelli A, Capelli G, Joachim A, Hinney B, Losson B, Kirkova Z, et al. Lungworms and gastrointestinal parasites of domestic cats: a European perspective. Int J Parasitol. 2017;47:517-28.

3. Cavalera MA, Schnyder M, Gueldner EK, Furlanello T, latta R, Brianti E, et al. Serological survey and risk factors of Aelurostrongy/us abstrusus infection among owned cats in Italy. Parasitol Res. 2019;118:2377-82.

4. Traversa D, Morelli S, Cassini R, Crisi PE, Russi I, Grillotti E, et al. Occurrence of canine and feline extra-intestinal nematodes in key endemic regions of Italy. Acta Trop. 2019;193:227-35.

5. Riggio F, Mannella R, Ariti G, Perrucci S. Intestinal and lung parasites in owned dogs and cats from central Italy. Vet Parasitol. 2013;193(1-3):78-84.

6. Sauda F, Malandrucco L, De Liberato C, Perrucci S. Gastrointestinal parasites in shelter cats of central Italy. Vet Parasitol Reg Stud Rep. 2019;18:100321.

7. Cringoli G, Maurelli MP, Levecke B, Bosco A, Vercruysse J, Utzinger J, Rinaldi L. The Mini-FLOTAC technique for the diagnosis of helminth and protozoan infections in humans and animals. Nat Protoc. 2017:12(9):1723-32.

8. Amadesi A, Bosco A, Rinaldi L, Cringoli G, Claerebout E, Maurelli MP. Cattle gastrointestinal nematode egg-spiked faecal samples: high recovery rates using the Mini-FLOTAC technique. Parasit Vectors. 2020;13(1):230

9. Catalano S, Symeou A, Marsh KJ, Borlase A, Léger E, Fall CB, et al. MiniFLOTAC as an alternative, non-invasive diagnostic tool for Schistosoma mansoni and other trematode infections in wildlife reservoirs. Parasit Vectors. 2019:12(1):439.

10. Ianniello D, Pepe P, Alves LC, Ciuca L, Maurelli MP, Amadesi A, et al. Why use the Mini-FLOTAC to detect metastrongyloid larvae in dogs and cats? Acta Parasitol. 2020;65(2):546-9.

11. Nápravníková J, Petrtýl M, Stupka R, Vadlejch J. Reliability of three common fecal egg counting techniques for detecting strongylid and ascarid infections in horses. Vet Parasitol. 2019;272:53-7.

12. Morandi B, Greenwood SJ, Conboy GA, Galuppi R, Poglayen G, VanLeeuwen JA. Endoparasites in dogs and cats diagnosed at the Veterinary Teaching Hospital (VTH) of the University of Prince Edward Island between 2000 and 2017. A large-scale retrospective study. Prev Vet Med. 2020:175:104878.
13. Sweet S, Szlosek D, McCrann D, Coyne M, Kincaid D, Hegarty E. Retrospective analysis of feline intestinal parasites: trends in testing positivity by age, USA geographical region and reason for veterinary visit. Parasit Vectors. 2020;13(1):473.

14. Maurelli MP, Dourado Martins OM, Morgan ER, Charlier J, Cringoli G, et al. A qualitative market analysis applied to Mini-FLOTAC and FillFLOTAC for diagnosis of helminth infections in ruminants. Front Vet Sci. 2020;7:580649

15. Soulsby ELJ. Helminth, arthropods, and protozoa of domesticated animals. 7th ed. London: Bailliere Tindal; 1982.

16. Bowman DD. Georgi's parasitology for veterinarians. 6th ed. Philadelphia: W. B. Saunders Company; 1995. p. 295-6.

17. Varcasia A, Brianti E, Tamponi C, Pipia AP, Cabras PA, Mereu M, et al. Simultaneous infection by four feline lungworm species and implications for the diagnosis. Parasitol Res. 2015;114(1):317-21.

18. Brianti E, Giannetto S, Dantas-Torres F, Otranto D. Lungworms of the genus Troglostrongylus (Strongylida: Crenosomatidae): neglected parasites for domestic cats. Vet Parasitol. 2014;202(3-4):104-12.

19. Castor EDC. Castor electronic data capture. 2019. https://castoredc.com. Accessed 10 Oct 2020.

20. Morgan ER, Azam D, Pegler K. Quantifying sources of environmental contamination with Toxocara spp. eggs. Vet Parasitol. 2013;193(4):390-7.

21. Azam D, Ukpai OM, Said A, Abd-Allah GA, Morgan ER. Temperature and the development and survival of infective Toxocara canis larvae. Parasitol Res. 2012;110(2):649-56.

22. Epe C. Intestinal nematodes: biology and control. Vet Clin North Am Small Anim Pract. 2009;39(6):1091-107.

23. Robertson ID, Thompson RC. Enteric parasitic zoonoses of domesticated dogs and cats. Microbes Infect. 2002;4(8):867-73.

24. Gueldner EK, Gilli U, Strube C, Schnyder M. Seroprevalence, biogeographic distribution and risk factors for Aelurostrongylus abstrusus infections in Swiss cats. Vet Parasitol. 2019;266:27-33.

25. Tamponi C, Varcasia A, Brianti E, Pipia AP, Frau V, Pinna Parpaglia ML, et al. New insights on metastrongyloid lungworms infecting cats of Sardinia, Italy. Vet Par. 2014;203(1-2):222.

26. Cooper AR, Nixon E, Rose Vineer H, Abdullah S, Newbury H, Wall R. Fleas infesting cats and dogs in Great Britain: spatial distribution of infestation risk and its relation to treatment. Med Vet Entomol. 2020;34(4):452-8.

27. Nardoni S, Ebani VV, Fratini F, Mannella R, Pinferi G, Mancianti F, et al. Malassezia, mites and bacteria in the external ear canal of dogs and cats with otitis externa. Slov Vet Res. 2014;51(3):113-8.

\section{Publisher's Note}

Springer Nature remains neutral with regard to jurisdictional claims in published maps and institutional affiliations.
Ready to submit your research? Choose BMC and benefit from:

- fast, convenient online submission

- thorough peer review by experienced researchers in your field

- rapid publication on acceptance

- support for research data, including large and complex data types

- gold Open Access which fosters wider collaboration and increased citations

- maximum visibility for your research: over $100 \mathrm{M}$ website views per year

At BMC, research is always in progress.

Learn more biomedcentral.com/submissions 